\title{
Copulatory behavior of Syrian golden hamsters in a one-male two-female test situation
}

\author{
DONALD A. DEWSBURY, DAVID L. LANIER, and JOSEPH M. OGLESBY \\ University of Florida, Gainesville, Florida 32611
}

\begin{abstract}
Eighteen male hamsters each completed two tests of copulatory behavior: one with one female present and one with two females. Males displayed only relatively small, quantitative alterations of their typical copulatory pattern in the two-female vs. the one-female situation. All males mated with both females. Shifts between females tended to occur at predictable times-particularly after ejaculations and those long intromissions that did not immediately precede ejaculations. When mating in the two-female situation, males sired more offspring than with one female. Females in the two-female situation received less copulatory stimulation than in the one-female situation, but there were no detectable deleterious effects of this reduced stimulation on reproduction. Should copulation occur between one male and two females in the natural habitat, it would appear to be of reproductive advantage to the male, with no detriment to the female.
\end{abstract}

Most laboratory tests of copulatory behavior are conducted with a single male-female pair mating at a time. Although such tests remove copulatory interactions from what may be their normal social context, they have been very useful in the investigation of a variety of problems. Various workers have noted that a truly comprehensive understanding of copulatory behavior can be gained only with studies in more complex social situations (e.g., Itzkowitz, 1974; McFarland \& Nunez, 1978). As a result, studies have been conducted of the copulatory behavior of various species in both multimale (Dewsbury, 1976, 1979; Evans \& Dewsbury, 1979; Larsson, 1956) and multifemale (Krames \& Mastromatteo, 1973; Tiefer, 1969) situations. The present paper reports the results of the first study of Syrian golden hamsters (Mesocricetus auratus) in a multifemale situation.

There are several sets of questions that can be asked when copulatory behavior is studied in complex social situations (i.e., when more than one male and one female are present). One can first ask whether the fundamental, species-typical copulatory pattern will be altered. Different species of muroid rodents display a variety of alternative patterns that can be treated as qualitatively distinct (Dewsbury, 1975). Most of these patterns have been studied only in rather simple situations; their generalizability to more complex situations has not often been tested. As normative data have been reported for the various measurable parameters of copulatory behavior in different species mating in one-male, one-female tests (see Dewsbury, 1975), one can investigate the extent of quantitative alteration of such parameters

Supported by Grant BNS78-05173 from the National Science Foundation. in more complex situations. From social facilitation theory (e.g., Zajonc, 1965), one might expect an overall acceleration of copulatory behavior in complex situations. In general, such alterations of copulatory behavior as have been reported have been statistically significant, but not major, changes in various parameters of copulatory behavior (Dewsbury, 1976, 1979; Evans \& Dewsbury, 1979; Krames \& Mastromatteo, 1973; Larsson, 1956; Tiefer, 1969). Several studies have indicated that a change of female after a male ceases mating can produce a resumption of copulatory behavior, the "Coolidge effect" (e.g., Fisher, 1962; Wilson, Kuehn, \& Beach, 1963).

A second set of questions that can be asked in studies of the multifemale situation relates to the distribution of the male's copulations. One can first ask whether the male will mate with all females or with just one. Confronted by two females in a laboratory test situation, a male rhesus monkey (Macaca mulatta) typically copulates with one "favorite", while both he and the favorite threaten the second female (Herbert, 1968). By contrast, laboratory rats (Rattus norvegicus) typically mate with all females present (Krames \& Mastromatteo, 1973; Tiefer, 1969). If the male does mate with more than one female, one can investigate the pattern according to which the male distributes those copulations. For example, Krames and Mastromatteo (1973) reported that male rats switched females between their last preejaculatory intromission and their ejaculation on $50 \%$ or more of their opportunities. Other details of such distribution also can be studied.

A third set of questions relates to the consequences of both the complex situation per se and of the particular distribution of the male's copulations for 
successful reproduction, from the standpoint of both male and female. Because most laboratory tests of copulation are conducted with females in hormoneinduced estrus, using an effective contraceptive dose, litters are not observable. By mating females in naturally occurring estrus, however, the offspring resulting from different tests can be counted. Thus, behavior can be related to differential reproduction. For example, it has been shown that increasing the ratio of ewes per ram in sheep (Ovis aries) has generally deleterious effects on copulatory behavior and reproduction (Allison, 1977). Such data bring studies of copulatory behavior into the realm of "mating strategies" and close to the context of contemporary sociobiology (e.g., Wilson, 1975).

In studies of laboratory rats in multifemale situations, it has been found that males often appear to copulate with whatever female happens to move past them as the males are ready to mount, thus producing an essentially random distribution of copulations (Krames \& Mastromatteo, 1973; Tiefer, 1969). There is reason to expect that different results might be generated from a similar study with hamsters. While female rats typically display much activity between copulations (e.g., Dewsbury, 1967), female hamsters hold the lordotic position long enough to permit multiple intromissions and multiple ejaculations with the female remaining in lordosis throughout (Bunnell, Boland, \& Dewsbury, 1977). As females are much less mobile, male hamsters may discriminate between two females more readily than do male rats, as hamsters have a much more clearcut basis for choice.

The distribution of copulations may be more critical for successful reproduction in hamsters than in rats. With young female rats mating in cycling estrus, a single ejaculation is sufficient for essentially maximal levels of pregnancy (Wilson, Adler, \& LeBoeuf, 1965). Thus, a female need receive only a relatively small proportion of a male's copulations for successful pregnancy. By contrast, young female hamsters have a much higher stimulus requirement (Lanier, Estep, \& Dewsbury, 1975). As a consequence, there is a more substantial danger that a male may "spread himself too thin," by delivering too few copulations to some females, thus providing too little stimulation for pregnancy.

All three sets of questions are addressed in the present study.

\section{METHOD}

\section{Subjects}

The subjects were 24 male and 38 female Syrian golden hamsters, Mesocricetus auratus, of which 18 males and 33 females contributed to the final data set. All were of the outbred Lak:LVG (SYR) strain, purchased from Charles River Lakeview, Wilmington, Mass., and at least 90 days of age at the time of testing. The animals were housed in standard Wahmann hanging cages with continuous access to Purina Lab Chow and water. A reversed 16L:8D photoperiod was used. All animals had been pretested for fertility by mating them and permitting them to sire or bear one litter.

\section{Apparatus}

Tests were conducted in a ventral view apparatus, with the mating chamber consisting of a Plexiglas cylinder, $51 \mathrm{~cm}$ in diameter and $62 \mathrm{~cm}$ high. This cylinder was placed on a glass floor, under which a mirror was mounted at a $45^{\circ}$ angle to permit observation of penile insertions. Data were recorded on an Esterline-Angus operations recorder.

\section{Procedure}

Each male received two tests of copulatory behavior-one in the one-female (1F) condition and one in the two-female (2F) condition. Eleven males completed the $2 F$ test first and seven males completed the $1 F$ test first. At least 9 days intervened between successive tests for each male.

Estrous stages were judged daily before tests and for 8 days after testing with extra-vaginal examinations using the method described by Orsini (1961). Tests were conducted in dim white light during the dark phase of the diurnal cycle. Males were introduced into the test arenas $10 \mathrm{~min}$ before the females. Tests were initiated with the introduction of one or two females, depending on the condition. In the two-female condition, the females were identified by short black marks made with a marking pen near either a foreleg or a hindleg. If copulation was initiated, tests were continued until the male attained a satiety criterion of 15 min with no intromissions (Beach \& Rabedeau, 1959).

If, after testing, the female returned to the estrous condition within 8 days, she was considered not pregnant and returned to the subject pool. If she failed to return to estrus, a metal pan with San-i-cel bedding, was inserted into the hanging cage prior to the anticipated parturition in order to provide a solid floor for the litter. The pups in each litter were counted on the day of parturition.

\section{Measure}

Four primary events characterize the copulatory behavior of male hamsters and are defined by Bunnell et al. (1977). During intromissions (or more correctly, short intromissions), the male mounts the female, gains vaginal insertion, delivers a single intravaginal thrust, but does not ejaculate. On mounts, the male mounts the female, but fails to gain insertion. During ejaculation, the male mounts the female, gains vaginal penetration, and ejaculates. Mounts, short intromissions, and ejaculations are organized into "series," with each series terminated by an ejaculation. After completing a number of such series, males display a shift in pattern. Long intromissions, similar to short intromissions but of longer duration and including intravaginal thrusting, are interspersed with ejaculations in a pattern less orderly than the organized series.

The following measures, defined by Bunnell et al. (1977), were used; mount latency (ML), intromission latency (IL), ejaculation latency (EL), intromission frequency (IF), mount frequency (MF), mean interintromission interval (MIII), postejaculatory interval (PEI), and total ejaculation frequency (EF). For measures that refer to a specific series, the series is designated when used (e.g., IF-2). In addition, the following measures were determined: the number of ejaculations attained during organized series, the number of ejaculations attained in association with long intromissions, the number of long intromissions, the total number of thrusts on all long intromissions, and the time elapsing from the first long intromission to the last.

\section{RESULTS}

The results can be examined with respect to both behavior and successful reproduction from the per- 
Table 1

Selected Measures of Male Copulatory Behavior as a Function of Condition

\begin{tabular}{|c|c|c|c|c|}
\hline \multirow[b]{3}{*}{ Measure } & \multicolumn{4}{|c|}{ Condition } \\
\hline & \multicolumn{2}{|c|}{ One Female } & \multicolumn{2}{|c|}{ Two Females } \\
\hline & Mean & SE & Mean & SE \\
\hline Mount Latency* & 58.8 & 14.4 & $81.2 \dagger$ & 8.4 \\
\hline Intromission Latency* & 74.2 & 14.9 & 103.4 & 11.1 \\
\hline Ejaculation Latency-1* & 104.0 & 11.0 & 131.0 & 22.4 \\
\hline Intromission Frequency-1 & 10.4 & 1.4 & 8.7 & .8 \\
\hline Mount Frequency-1 & 3.4 & 1.0 & 7.0 & 2.8 \\
\hline Mean Interintromission Interval-1* & 10.9 & .9 & 16.0 & 3.7 \\
\hline Postejaculatory Interval-1* & 38.2 & 2.8 & 47.0 & 10.2 \\
\hline Total Ejaculation Frequency & 11.3 & 1.3 & 10.8 & 1.0 \\
\hline Ejaculation Frequency in Series & 7.0 & .3 & $7.4+\dagger$ & .4 \\
\hline Ejaculation Frequency with Long Intromissions & 4.3 & 1.1 & 3.4 & .9 \\
\hline Number of Long Intromissions & 32.1 & 3.2 & 31.7 & 2.5 \\
\hline Number of Thrusts on Long Intromissions & 268.4 & 26.1 & 327.2 & 35.2 \\
\hline Time Span for Long Intromissions* & 1917.6 & 197.1 & $2585.9 * *$ & 234.6 \\
\hline
\end{tabular}

*In seconds. $\quad t p<.05$ for running order. $\quad t+p<.05$ for condition.

spective of both the male and the female. There was little effect of the one-female vs. two-female condition on male copulatory behavior (see Table 1). There were no qualitative alterations in pattern in the two-female situation. Effects of the $1 F$ vs. the $2 F$ condition and of running order were assessed with two-way analyses of variance with repeated measures (Winer, 1962) for 28 measures characterizing the first four series and the long-intromission period. The only significant effects of condition were on the ejaculation frequency in series and the total time span of the long intromissions (see Table 1). The only significant effect of running order was on $\mathrm{ML}$, where the 2F-first males had a mean ML of $54.4 \mathrm{sec}$ compared to the 98.6 of the $1 F$-first males.

Males distributed their copulations to both females in all tests in the two-female condition. As two females were available for mating, one can determine which female received more and which fewer total intromissions, ejaculations, etc. Such data are summarized in Table 2 . The most notable feature of Table 2 is the lack of zeroes in the ranges. That is, every female in every $2 F$ test received at least one short intromission, two ejaculations, four long intromissions, and 37 thrusts on long intromissions. As the female receives one deep thrust on each short intromission and ejaculation, together with a variable number of thrusts on long intromissions, the sum of the number of short intromissions, ejaculations, and long-intromission thrusts provides a measure of the total number of thrusts received. Each female received at least 52 thrusts. For most measures, there was approximately a $2: 1$ ratio of stimulation, with $2 / 3$ of the copulations delivered to one female and $1 / 3$ to the other.

In order to mate with both females, the male must shift from one to the other on some occasions; the position of such shifts in the orderly copulatory pattern are summarized in Table 3. Males shifted from one female to the other on $8 \%-15 \%$ of the possible occasions between successive short intromissions, between short intromissions and ejaculations, and between long intromissions and ejaculations. Thus, they tended to continue mating with the same female on approximately $90 \%$ of these occasions. By contrast, males tended to shift more frequently between ejaculations and short intromissions, between ejaculations and long intromissions, and between two successive long intromissions $(34 \%$ $45 \%$ ). Thus, whereas males were generally not likely to shift females after short intromissions and just before ejaculations, they were more likely to shift

Table 2

Distribution by Male of Copulations to Two Females

\begin{tabular}{|c|c|c|c|c|}
\hline \multirow[b]{2}{*}{ Measure } & \multicolumn{2}{|c|}{ High Female } & \multicolumn{2}{|c|}{ Low Female } \\
\hline & Mean & Range & Mean & Range \\
\hline Total Number of Ejaculations & 7.2 & $3-17$ & 3.3 & $2-7$ \\
\hline Total Number of Intromissions & 16.3 & $9-30$ & 7.1 & $1-12$ \\
\hline Total Number of Long Intromissions & 19.2 & 4-34 & 11.3 & $4-21$ \\
\hline Number of Long Intromission Thrusts & 196.0 & $51-380$ & 121.0 & $37-283$ \\
\hline Total Number of Thrusts & 215.6 & $66-384$ & 135.4 & 52.296 \\
\hline
\end{tabular}


Table 3

Pattern of Shifts by Male Hamsters in Two-Female Tests

\begin{tabular}{lccr}
\hline \multicolumn{1}{c}{ Transition } & Shifts & Opportunities & Percent Shifts \\
\hline Intromission to Ejaculation & 11 & 131 & 8.4 \\
Intromission to Intromission & 31 & 290 & 10.7 \\
Long Intromission to Ejaculation & 9 & 60 & 15.0 \\
Ejaculation to Intromission & 39 & 113 & 34.5 \\
Long Intromission to Long Intromission & 164 & 475 & 34.5 \\
Ejaculation to Long Intromission & 33 & 74 & 44.6 \\
\hline
\end{tabular}

after ejaculations and before long intromissions.

In their study of rats in a two-female situation, Krames and Mastromatteo (1973) found few significant "runs" in which a male mated with a single female. By contrast, there were many long runs in the present data. In 13 of the 18 tests, there was at least one run in which a male completed three complete successive ejaculatory series without shifting females. In 5 tests, there were such runs of five or more series, including one run of eight consecutive series.

As a result of their pattern of distributing copulations, males were successful in producing more of fspring when mating in the $2 \mathrm{~F}$ condition than in the $1 \mathrm{~F}$ condition. One complication in interpreting data is that on eight occasions a litter was delivered, but was destroyed prior to counting (see Table 4). One might argue that these instances should be counted as cases of zero production of offspring, as no live pups were produced. The case for such treatment is strengthened by the demonstration that in hamsters inadequate vaginal stimulation at the time of mating can produce abnormalities at parturition (Diamond, 1972). Alternatively, one might argue that such cases may be an artifact of captivity and that cases with such litter destruction should be considered as of indeterminate result and thus deleted from analysis. The results are essentially identical with either treatment. When all data are included, the 18 males produced a mean of 4.7 pups in the $1 \mathrm{~F}$ condition versus 12.8 in the $2 F$ condition, a significant difference $[t(17)=3.50, p<.01]$. When cases with litter destruction were deleted, 12 males produced a mean of 5.8 pups in the $1 \mathrm{~F}$ condition and 16.1 in the $2 \mathrm{~F}$ condition $[\mathrm{t}(11)=3.39, \mathrm{p}<.01]$.

Table 4

Consequences of One-Female vs. Two-Female Condition for Reproduction

Condition

\begin{tabular}{lrrrrr} 
& \multicolumn{4}{c}{ Condition } \\
\cline { 5 - 6 } \multicolumn{1}{c}{ Outcome } & \multicolumn{1}{c}{ One Female } & Two Females \\
\cline { 2 - 3 } \cline { 5 - 6 } & $\mathrm{N}$ & Percent & & N & Percent \\
\hline Litter Delivered Intact & 11 & 61 & 24 & 67 \\
Litter Delivered but Destroyed & 2 & 11 & 6 & 17 \\
Not Pregnant & 3 & 17 & 2 & 6 \\
Pseudopregnant & 2 & 11 & 4 & 11 \\
\hline
\end{tabular}

The data will now be viewed from the perspective of individual females. Females received less copulatory stimulation when mating in the $2 \mathrm{~F}$ than in the $1 \mathrm{~F}$ condition (see Table 5). When mating in the $2 \mathrm{~F}$ condition, females endured a longer latency to the first mount and intromission and received significantly fewer copulations in every category presented in Table 5 .

Although females received less copulatory stimulation in the $2 \mathrm{~F}$ condition, there was no evidence that the lack of stimulation had a detrimental effect on reproduction. As seen in Table 4, the percent of females delivering intact litters, delivering litters that were destroyed, not pregnant, and pseudopregnant did not differ between the $1 F$ and $2 F$ conditions. Further, as seen in Table 5, there were no significant differences between conditions with respect to the litter sizes of females that did actually deliver litters whose numbers could be accurately counted.

Examination of data from individual females revealed only a weak association between amount of stimulation received and reproduction. Overall, 10 females received fewer than 100 thrusts ( 2 females in the $1 \mathrm{~F}$ condition and 8 females in the $2 \mathrm{~F}$ condition). Seven of those females delivered intact litters, with a typical distribution of litter sizes, and three were pseudopregnant. The only group with consistently fewer thrusts than the others was the pseudopregnant females in the $2 F$ condition, which received 66 , 72,78 , and 102 thrusts. The two pseudopregnant females in the $1 \mathrm{~F}$ condition, however, received 280 and 317 thrusts. The five females becoming neither pregnant nor pseudopregnant received a range of 217-351 thrusts.

\section{DISCUSSION}

From the standpoint of the male hamster, mating in the two-female condition produced little alteration of the typical pattern, a distribution of copulations delivered to both females, and a significant increase in overall reproduction relative to the one-female condition. From the standpoint of the female, the $2 F$ condition resulted in reduced copulatory stimulation, but with no constant decrease in reproduction.

As has been reported for laboratory rats (Tiefer, 1969), the availability of more than one female produced no qualitative alteration in copulatory pattern and no increase in the total number of 
Table 5

Copulatory Behavior and Litter Size for Female Hamsters in the One-Female and Two-Female Conditions

\begin{tabular}{|c|c|c|c|}
\hline \multirow[b]{2}{*}{ Measure } & \multicolumn{2}{|c|}{ Condition } & \multirow[b]{2}{*}{$\mathrm{t}$} \\
\hline & One Female & Two Females & \\
\hline Mount Latency & 63.1 & 164.9 & $2.27 *$ \\
\hline Intromission Latency & 78.2 & 198.6 & $2.60^{*}$ \\
\hline Total Ejaculation Frequency & 10.4 & 5.2 & $4.68^{* *}$ \\
\hline Ejaculation Frequency in Series & 6.8 & 3.6 & $5.57 * *$ \\
\hline Ejaculation Frequency with Long Intromissions & 3.7 & 1.6 & $2.50^{*}$ \\
\hline Total Number of Intromissions & 24.5 & 11.7 & $5.62 * *$ \\
\hline Number of Long Intromissions & 30.8 & 15.3 & $5.34 * *$ \\
\hline Number of Thrusts on Long Intromissions & 256.8 & 158.5 & $3.42 * *$ \\
\hline Total Number of Thrusts & 291.7 & 175.5 & $4.03^{* *}$ \\
\hline Litter Size & 7.6 & 9.6 & 1.59 \\
\hline
\end{tabular}

Note-Degrees of freedom $=33$ for litter size and 52 for all other measures. $\quad *_{p}<.05 . \quad *^{*} p<.001$.

ejaculations attained. Tiefer interpreted this overall consistency of pattern as reflecting "a strong internal programme" in the male (p. 720). Hamsters would appear to have a similarly "strong internal programme" with the occurrence of sexual satiety, operationally defined, a function of that program rather than of characteristics of a single female. Although Tiefer reported a number of quantitative changes in copulatory behavior in the first series of rats in a multifemale situation, no such effects were found in the present study with hamsters.

Unlike rhesus monkeys, but like laboratory rats, male hamsters reliably copulated with both females in the multifemale situation. However, while the distribution of copulations in rats appears to be random (Krames \& Mastromatteo, 1973), hamsters tended to deliver runs of copulations to each of the two females. Males were especially likely to shift females after ejaculations and after those long intromissions that did not immediately precede an ejaculation. Overall, males delivered ejaculations to the same female as that with which they had just intromitted on $90 \%$ of the occasions. This, too, contrasts with rats, which shift females just before ejaculation on $50 \%$ or more of their opportunities (Krames \& Mastromatteo, 1973). The differences between male rats and hamsters probably reflect, at least in part, the difference in activity during intervals between successive copulations in female rats, which are quite active, vs. female hamsters, which are inactive (Bunnell et al., 1977; Dewsbury, 1967). Rather than being confronted with two or more active, darting females as in rats, the male hamster about to intromit is confronted with two immobile females, one of which is generally immediately in front of the location in which he has just completed genital grooming.

Because female golden hamsters require more copulatory stimulation for pregnancy and parturition than do rats (Diamond, 1972; Lanier et al., 1975; Wilson et al., 1965), there is a risk that, in the $2 \mathrm{~F}$ condition, a male may deliver too few copulations to result in successful pregnancy and parturition. Such was not the case; confronted with two females, males sired significantly more offspring than with just one, even though they did no more copulating.

For females, tests in the $2 \mathrm{~F}$ condition resulted in less copulatory stimulation than in the $1 \mathrm{~F}$ condition, but with no detrimental effects on reproduction. It is likely that the long intromissions play an important role in ensuring that both females receive sufficient copulatory stimulation for pregnancy. One function for long intromissions appears to lie in prolonging copulatory activity in a manner that reduces the effects of sperm competition in tests in which two males mate successively with a single female (Oglesby, Lanier, $\&$ Dewsbury, Note 1). In addition, the long intromissions appear to permit a male to deliver substantial amounts of copulatory stimulation to two females, within possible limits of sperm stores.

The relevance of these experimental data to the natural condition is not clear. The data suggest that natural selection should act to favor males that would mate with two females, but not to select against females mating with more than one male. It would be interesting to increase the female/male ratio further to determine the point at which decreased stimulation received by some females would begin to be detrimental to reproduction. Although there are a few evidences in seminatural studies (e.g., Goldman \& Swanson, 1975), hamsters are generally believed to be solitary in the natural habitat (Murphy, 1971). It is not clear how often, if at all, a male would have the opportunity to copulate with more than one female. In order to determine this, one would have to study the movement patterns of males and females in nature during the time of the female's estrus. Should males move to females' burrows, multifemale interactions would be unlikely. Should estrous females move toward males' burrows, however, arrival of more than one female may be more frequent. Female hamsters are known to be more active during their proestrous and estrous days than at other times. This 
is true of activity as measured both in running wheels and with home-cage observations (Richards, 1966). In addition, activity decreases immediately after mating. Such data suggest the possibility that in their natural habitat females may indeed move away from their isolated burrows when in estrus. The present data, collected under laboratory conditions, suggest that if this occurs and a male mates with two females, the male would benefit with an increased Darwinian fitness, with no detriment to the female.

\section{REFERENCE NOTE}

1. Oglesby, J. M., Lanier, D. L., \& Dewsbury, D. A. The role of prolonged copulatory behavior in facilitating reproductive success in male Syrian golden hamsters (Mesocricetus auratus) in a competitive mating situation. Manuscript in preparation.

\section{REFERENCES}

Allisox. A. J. Flock mating in sheep. Il. Effect of number of ewes per ram on mating behavior, and fertility of two-tooth and mixed-age Romney ewes run together. New Zealand Journal of Agricultural Research, 1977, 20, 123-128.

Bfach. F. A.. \& Rabedeau, R. G. Sexual exhaustion and recovery in the male hamster. Journal of Comparative and Physinlogical Psychology, 1959, 52, 56-61.

Bi vift.l, B. N., Boland, B. D., \& Dewsbury, D. A. Copulatory behavior of golden hamsters (Mesocricetus auratus). Behaviour, 1977, 61, 180-206.

DeWSBLRY, D. A. A quantitative description of the behavior of rats during copulation. Behaviour, 1967, 29, 154-178.

DewsBCRY, D. A. Diversity and adaptation in rodent copulatory behavior. Science, 1975, 190, 947-954.

Dewsbury, D. A. Copulatory behavior of white-footed mice (Peromyscus leucopus) in a multimale situation. Bulletin of the Psychonomic Society, 1976, 7, 340-342.

Dewsbury, D. A. Copulatory behavior of deermice (Peromyscus maniculatus): II. A study of some factors regulating the fine structure of behavior. Journal of Comparative and Physiological Psychology, 1979, 93, 161-177.

DiAmovD. M. Vaginal stimulation and progesterone in relation to pregnancy and parturition. Biology of Reproduction, 1972, 6, 281-287.

Evans, R. L., \& Dewsbury, D. A. Copulatory behavior of prairie voles (Microtus ochrogaster) in a two-male situation. Behavioral Biology, 1979, 24, 498-508.

Fisher, A. E. Effects of stimulus variation on sexual satiation in the male rat. Journal of Comparative and Phisiological Psychology, 1962, 55, 614-620.

Goldman, L., \& Swanson, H. Population control in confined colonies of golden hamsters (Mesocricelus auratus Waterhouse). Zeitschrifı für Tierpsychologie, 1975, 37, 225-236.

Herbert, J. Sexual preference in the rhesus monkey Macaca mulatta in the laboratory. Animal Behavior, 1968, 16, 120-128.

ITzkowitz, M. The effects of other fish on the reproductive behavior of the male Cyprinodon variegatus (Pisces: Cyprinodontidae). Behaviour, 1974, 48, 1-22.

Krames, L., \& Mastromatteo, L. A. Role of olfactory stimuli during copulation in male and female rats. Journal of Comparative and Physiological Psychology, 1973, 85, 528-535.

Lanier, D. L., Estep, D. Q., \& Dewsbury, D. A. Copulatory behavior of golden hamsters: Effects on pregnancy. Physiology \& Behavior, 1975, 15, 209-212.

LARSSON, K. Conditioning and sexual behavior in the male albino rat. Acta Psychologica Gothoburgensia, 1956, 1, 1-269.

McFarland, D., \& Nunez, A. T. Systems analysis and sexual behavior. In J. B. Hutchison (Ed.), Biological determinants of sexual behavior. New York: Wiley, 1978.

MURPHY, M. R. Natural history of the Syrian golden hamstera reconnaissance expedition. American Zoologist, 1971, 11, 632

Orsini, M. W. The external vaginal phenomena characterizing the stages of the estrous cycle, pregnancy, pseudopregnancy, lactation, and the anestrous hamster, Mesocricetus auratus Waterhouse. Proceedings of the Animal Care Panel, 1961, 11, 193-206.

Richands, M. P. M. Activity measured by running wheels and observation during the oestrous cycle, pregnancy and pseudopregnancy in the golden hamster. Animal Behaviour 1966, 14, 450-458.

TIEFER, L. Copulatory behavior of male Rattus norvegicus in a multiple female exhaustion test. Animal Behaviour, 1969, 17, 718-721.

Wilson, E. O. Sociobiology: The new synthesis. Cambridge, Mass: Harvard University Press, 1975.

Wilson, J. R., Adler, N., \& LeBoeuf, B. The effects of intromission frequency on successful pregnancy in the female rat. Proceedings of the National Academy of Sciences, U.S.A., $1965,53,1392-1395$.

Wilson, J. R., Kuehn, R. E., \& Beach, F. A. Modification in the sexual behavior of male rats produced by changing the stimulus female. Journal of Comparative and Physiological Psychology, 1963, 56, 636-644.

Winer, B. J. Statistical principles in experimental design. New York: McGraw-Hill, 1962.

ZAJonc, R. B. Social facilitation. Science, 1965, 149, 269-274.

(Received for publication December 13, 1978; revision accepted April 10, 1979.) 